\title{
Indiana Traffic Signal Hi Resolution Data Logger Enumerations
}

August 2020

Howell Li, Purdue University

Alexander M. Hainen, University of Alabama

James R. Sturdevant, Indiana Department of Transportation

Travis Atkison, University of Alabama

Sufian Talukder, University of Alabama

Jijo K. Mathew, Purdue University

Darcy M. Bullock, Purdue University

Daniel Nelson, Siemens Industry Inc.

Donald M. Maas, Jr., McCain Inc.

Joshua Fink, Econolite Group Inc.

Tom Stiles, Intelight Inc. 


\section{Abstract}

This document defines the enumerations used to encode events at a 100 millisecond resolution that occur on a traffic signal controller with high resolution data loggers. The enumerations definitions were developed as part of a multi-state pooled fund study, TPF-5(377), conducted in 2018-2019 that conducted a series of stakeholder engagements and panel meetings.

\section{Background}

This work enhancing the signal performance measures was organized through the United States Department of Transportation's Pooled Fund Program TPF-5(377) sponsored by the following state Departments of Transportation: California, Connecticut, Georgia, Indiana, Minnesota, North Carolina, Ohio, Pennsylvania, Texas, Utah, Wisconsin, the Federal Highway Administration and the City of College Station. This document is intended to replace the enumeration document developed in 2012.

Sturdevant, J. R., T. Overman, E. Raamot, R. Deer, D. Miller, D. M. Bullock, C. M. Day, T. M. Brennan, H. Li, A. Hainen, and S. M. Remias. Indiana Traffic Signal Hi Resolution Data Logger Enumerations. , Indiana Department of Transportation and Purdue University, West Lafayette, Indiana, 2012. https://doi.org/10.4231/K4RN35SH

A summary of past high-resolution traffic signal performance measures can be found at:

Day, C. M., D. M. Bullock, H. Li, S. M. Remias, A. M. Hainen, R. S. Freije, A. L. Stevens, J. R. Sturdevant, and T. M. Brennan. Performance Measures for Traffic Signal Systems: An Outcome-Oriented Approach. Purdue University, West Lafayette, Indiana, 2014. https://doi.org/10.5703/1288284315333

Day, C. M., D. M. Bullock, H. Li, S. Lavrenz, W. B. Smith, and J. R. Sturdevant. Integrating Traffic Signal Performance Measures into Agency Business Processes. Purdue University, West Lafayette, Indiana, 2015. https://doi.org/10.5703/1288284316063 


\section{Enumerations}

\begin{tabular}{|c|c|c|c|}
\hline $\begin{array}{l}\text { Event } \\
\text { Code }\end{array}$ & Event Descriptor & Parameter & Description \\
\hline \multicolumn{4}{|c|}{ Active Phase Events: } \\
\hline 0 & Phase On & Phase \# (1-255) & $\begin{array}{l}\text { Set when NEMA Phase On becomes active, } \\
\text { either upon start of green or walk interval, } \\
\text { whichever occurs first. }\end{array}$ \\
\hline 1 & Phase Begin Green & Phase \# (1-255) & $\begin{array}{l}\text { Set when either solid or flashing green } \\
\text { indication has begun. Do not set repeatedly } \\
\text { during flashing operation. }\end{array}$ \\
\hline 2 & Phase Check & Phase \# (1-255) & $\begin{array}{l}\text { Set when a conflicting call is registered } \\
\text { against the active phase. (Marks beginning } \\
\text { of MAX timing) }\end{array}$ \\
\hline 3 & Phase Min Complete & Phase \# (1-255) & Set when phase min timer expires. \\
\hline 4 & Phase Gap Out & Phase \# (1-255) & $\begin{array}{l}\text { Phase termination due to gap out } \\
\text { termination condition. Set once per phase } \\
\text { when phase gaps out but may not } \\
\text { necessarily occur upon phase termination. }\end{array}$ \\
\hline 5 & Phase Max Out & Phase \# (1-255) & $\begin{array}{l}\text { Set when phase MAX timer expires but may } \\
\text { not necessarily occur upon phase } \\
\text { termination due to last car passage or other } \\
\text { features. }\end{array}$ \\
\hline 6 & Phase Force Off & Phase \# (1-255) & $\begin{array}{l}\text { Set when phase force off is applied by the } \\
\text { coordinator to the active green phase. }\end{array}$ \\
\hline 7 & Phase Green Termination & Phase \# (1-255) & $\begin{array}{l}\text { Set when phase green indications are } \\
\text { terminated into either yellow change interval } \\
\text { or permissive (FYA) movement. }\end{array}$ \\
\hline 8 & $\begin{array}{l}\text { Phase Begin Yellow } \\
\text { Change }\end{array}$ & Phase \# (1-255) & $\begin{array}{l}\text { Set when phase yellow indication becomes } \\
\text { active and interval timer begins. }\end{array}$ \\
\hline 9 & $\begin{array}{l}\text { Phase End Yellow } \\
\text { Change }\end{array}$ & Phase \# (1-255) & $\begin{array}{l}\text { Set when phase yellow indication becomes } \\
\text { inactive. }\end{array}$ \\
\hline 10 & $\begin{array}{l}\text { Phase Begin Red } \\
\text { Clearance }\end{array}$ & Phase \# (1-255) & $\begin{array}{l}\text { Set only if phase red clearance is served. } \\
\text { Set when red clearance timing begins. }\end{array}$ \\
\hline 11 & $\begin{array}{l}\text { Phase End Red } \\
\text { Clearance }\end{array}$ & Phase \# (1-255) & $\begin{array}{l}\text { Set only if phase red clearance is served. } \\
\text { Set when red clearance timing concludes. } \\
\text { This may not necessarily coincide with } \\
\text { completion of the phase, especially during } \\
\text { clearance of trailing overlaps, red revert } \\
\text { timing, red rest, or delay for other ring } \\
\text { terminations. }\end{array}$ \\
\hline 12 & Phase Inactive & Phase \# (1-255) & $\begin{array}{l}\text { Set when the phase is no longer active } \\
\text { within the ring, including completion of any } \\
\text { trailing overlaps or end of barrier delays for } \\
\text { adjacent ring termination. }\end{array}$ \\
\hline
\end{tabular}




\begin{tabular}{|c|c|c|c|}
\hline 13 & Extension Timer Gap Out & Phase \# (1-255) & Set when phase extension timer gaps out. \\
\hline 14 & Phase Skipped & Phase \# (1-255) & $\begin{array}{l}\text { Set when phase in the programmed ring is } \\
\text { skipped for any reason. }\end{array}$ \\
\hline 15 & $\begin{array}{l}\text { Extension Timer } \\
\text { Reduction Start }\end{array}$ & Phase \# (1-255) & $\begin{array}{l}\text { Set when extension timer starts to reduce } \\
\text { (the time before reduction). }\end{array}$ \\
\hline 16 & $\begin{array}{l}\text { Extension Timer Minimum } \\
\text { Achieved }\end{array}$ & Phase \# (1-255) & $\begin{array}{l}\text { Set when extension timer minimum is } \\
\text { reached (after the time to reduce). }\end{array}$ \\
\hline 17 & Added Initial Complete & Phase \# (1-255) & Set when phase added initial timer expires. \\
\hline 18 & Next Phase Decision & Phase \# (1-255) & $\begin{array}{l}\text { Set when the controller determines a phase } \\
\text { will be next to begin green after the current } \\
\text { active phase(s) end red clearance. }\end{array}$ \\
\hline 19 & TSP Early Force Off & Phase \# (1-255) & $\begin{array}{l}\text { Set when TSP early force off is applied to an } \\
\text { active phase. }\end{array}$ \\
\hline 20 & Preemption Force Off & Phase \# (1-255) & $\begin{array}{l}\text { Set when controller applies preemption } \\
\text { force off to the active cycle. }\end{array}$ \\
\hline
\end{tabular}




\begin{tabular}{|c|c|c|c|}
\hline \multicolumn{4}{|c|}{ Active Pedestrian Events: } \\
\hline 21 & Pedestrian Begin Walk & Phase \# (1-255) & Set when walk indication becomes active. \\
\hline 22 & $\begin{array}{l}\text { Pedestrian Begin Change } \\
\text { Interval }\end{array}$ & Phase \# (1-255) & $\begin{array}{l}\text { Set when flashing don't walk indication } \\
\text { becomes active. }\end{array}$ \\
\hline 23 & $\begin{array}{l}\text { Pedestrian Begin Solid } \\
\text { Don't Walk }\end{array}$ & Phase \# (1-255) & $\begin{array}{l}\text { Set when don't walk indication becomes } \\
\text { solid (non-flashing) from either termination } \\
\text { of pedestrian change interval, or head } \\
\text { illumination after a pedestrian dark interval. }\end{array}$ \\
\hline 24 & Pedestrian Dark & Phase \# (1-255) & Set when the pedestrian outputs are set off. \\
\hline 25 & $\begin{array}{l}\text { Extended Pedestrian } \\
\text { Change Interval }\end{array}$ & Phase \# (1-255) & $\begin{array}{l}\text { Set when extended pedestrian change } \\
\text { interval is requested by pressing the } \\
\text { pedestrian push button for two (2) seconds. } \\
\text { See } 2009 \text { MUTCD Section } 4 \text { E.13 - } \\
\text { Accessible Pedestrian Signals and } \\
\text { Detectors - Extended Pushbutton Press } \\
\text { Features for more details. }\end{array}$ \\
\hline 26 & $\begin{array}{l}\text { Oversized Pedestrian } \\
\text { Served }\end{array}$ & Phase \# (1-255) & $\begin{array}{l}\text { Set when pedestrian phase is active beyond } \\
\text { pedestrian change interval or force off point. }\end{array}$ \\
\hline $27-30$ & $\begin{array}{l}\text { Pedestrian events } \\
\text { reserved for future use. }\end{array}$ & & \\
\hline \multicolumn{4}{|c|}{ Barrier / Ring Events: } \\
\hline 31 & Barrier Termination & Barrier \# (1-255) & $\begin{array}{l}\text { Set when all active phases become inactive } \\
\text { in the ring and cross barrier phases are next } \\
\text { to be served. }\end{array}$ \\
\hline 32 & FYA - Begin Permissive & FYA \# (1-255) & $\begin{array}{l}\text { Set when flashing yellow arrow becomes } \\
\text { active. }\end{array}$ \\
\hline 33 & FYA - End Permissive & FYA \# (1-255) & $\begin{array}{l}\text { Set when flashing yellow arrow becomes } \\
\text { inactive through either clearance of the } \\
\text { permissive movement or transition into a } \\
\text { protected movement. }\end{array}$ \\
\hline $34-40$ & $\begin{array}{l}\text { Barrier events reserve for } \\
\text { future use. }\end{array}$ & & \\
\hline
\end{tabular}




\begin{tabular}{|c|c|c|c|}
\hline \multicolumn{4}{|c|}{ Phase Control Events: } \\
\hline 41 & Phase Hold Active & Phase \# (1-255) & $\begin{array}{l}\text { Set when phase hold is applied by the } \\
\text { coordinator, preemptor, or external logic. } \\
\text { Phase does not necessarily need to be } \\
\text { actively timing for this event to occur. }\end{array}$ \\
\hline 42 & Phase Hold Released & Phase \# (1-255) & $\begin{array}{l}\text { Set when phase hold is released by the } \\
\text { coordinator, preemptor, or external logic. } \\
\text { Phase does not necessarily need to be } \\
\text { actively timing for this event to occur. }\end{array}$ \\
\hline 43 & Phase Call Registered & Phase \# (1-255) & $\begin{array}{l}\text { Call to service on a phase is registered by } \\
\text { vehicular demand. This event will not be set } \\
\text { if a recall exists on the phase. }\end{array}$ \\
\hline 44 & Phase Call Dropped & Phase \# (1-255) & $\begin{array}{l}\text { Call to service on a phase is cleared by } \\
\text { either service of the phase or removal of } \\
\text { call. }\end{array}$ \\
\hline 45 & $\begin{array}{l}\text { Pedestrian Call } \\
\text { Registered }\end{array}$ & Phase \# (1-255) & $\begin{array}{l}\text { Call to service on a phase is registered by } \\
\text { pedestrian demand. This event will not be } \\
\text { set if a recall exists on the phase. }\end{array}$ \\
\hline 46 & Phase Omit On & Phase \# (1-255) & $\begin{array}{l}\text { Set when phase omit is applied by the } \\
\text { coordinator, preemptor, or other dynamic } \\
\text { sources. Phase does not necessarily need } \\
\text { to be actively timing for this event to occur. } \\
\text { This event is not set when phase is removed } \\
\text { from the active sequence or other } \\
\text { configuration-level change has occurred. }\end{array}$ \\
\hline 47 & Phase Omit Off & Phase \# (1-255) & $\begin{array}{l}\text { Set when phase omit is released by the } \\
\text { coordinator, preemptor, or other dynamic } \\
\text { sources. Phase does not necessarily need } \\
\text { to be actively timing for this event to occur. } \\
\text { This event is not set when phase is added } \\
\text { from the active sequence or other } \\
\text { configuration-level change has occurred. }\end{array}$ \\
\hline 48 & Pedestrian Omit On & Phase \# (1-255) & $\begin{array}{l}\text { Set when pedestrian omit is applied by the } \\
\text { coordinator, preemptor, or other dynamic } \\
\text { sources. Phase does not necessarily need } \\
\text { to be actively timing for this event to occur. } \\
\text { This event is not set when phase is removed } \\
\text { from the active sequence or other } \\
\text { configuration-level change has occurred. }\end{array}$ \\
\hline 49 & Pedestrian Omit Off & Phase \# (1-255) & $\begin{array}{l}\text { Set when pedestrian omit is released by the } \\
\text { coordinator, preemptor, or other dynamic } \\
\text { sources. Phase does not necessarily need } \\
\text { to be actively timing for this event to occur. } \\
\text { This event is not set when phase is added } \\
\text { from the active sequence or other } \\
\text { configuration-level change has occurred. }\end{array}$ \\
\hline
\end{tabular}




\begin{tabular}{|l|l|l|l|}
\hline 50 & MAX 1 In-Effect & Phase \# (1-255) & $\begin{array}{l}\text { Set when maximum green (MAX 1) interval } \\
\text { is in-effect for the active phase. }\end{array}$ \\
\hline 51 & MAX 2 In-Effect & Phase \# (1-255) & $\begin{array}{l}\text { Set when maximum green (MAX 2) interval } \\
\text { is in-effect for the active phase. }\end{array}$ \\
\hline 52 & Dynamic MAX In-Effect & Phase \# (1-255) & $\begin{array}{l}\text { Set when dynamic max interval is in-effect } \\
\text { for the active phase. This event shall be } \\
\text { populated upon termination of MAX green } \\
\text { (MAX 1 or MAX 2) interval. }\end{array}$ \\
\hline 53 & Dynamic MAX Step Up & Phase \# (1-255) & $\begin{array}{l}\text { Set when dynamic max interval steps up for } \\
\text { the active phase (initially after two } \\
\text { consecutive phase max out events). }\end{array}$ \\
\hline 54 & $\begin{array}{l}\text { Dynamic MAX Step Down } \\
\text { Phase \# (1-255) }\end{array}$ & $\begin{array}{l}\text { Set when dynamic max interval steps down } \\
\text { for the active phase (initially after two } \\
\text { consecutive phase gap out events). }\end{array}$ \\
\hline 55 & $\begin{array}{l}\text { Advance Warning Sign } \\
\text { On }\end{array}$ & Phase \# (1-255) & Set when advance warning sign is on. \\
\hline 56 & $\begin{array}{l}\text { Advance Warning Sign } \\
\text { Off }\end{array}$ & Phase \# (1-255) & Set when advance warning sign is off. \\
\hline $57-60$ & $\begin{array}{l}\text { Phase Control Events } \\
\text { reserved for future use }\end{array}$ & \multicolumn{2}{|l}{} \\
\hline
\end{tabular}




\begin{tabular}{|c|c|c|c|}
\hline \multicolumn{4}{|c|}{ Overlap Events: } \\
\hline 61 & Overlap Begin Green & $\begin{array}{l}\text { Overlap \# (as number } \\
A=1 B=2 \text {, etc) }\end{array}$ & $\begin{array}{l}\text { Set when overlap becomes green. Do not } \\
\text { set repeatedly when overlap is flashing } \\
\text { green. Note that overlap colors are } \\
\text { consistent to the GYR intervals resultant } \\
\text { from the controller programming and may } \\
\text { not be indicative of actual signal head } \\
\text { colors. }\end{array}$ \\
\hline 62 & $\begin{array}{l}\text { Overlap Begin Trailing } \\
\text { Green (Extension) }\end{array}$ & $\begin{array}{l}\text { Overlap \# (as number } \\
A=1 B=2 \text {, etc) }\end{array}$ & $\begin{array}{l}\text { Set when overlap is green and extension } \\
\text { timers begin timing. }\end{array}$ \\
\hline 63 & Overlap Begin Yellow & $\begin{array}{l}\text { Overlap \# (as number } \\
A=1 B=2 \text {, etc) }\end{array}$ & $\begin{array}{l}\text { Set when overlap is in a yellow change } \\
\text { interval state. } \\
\text { Note that overlaps which drive yellow field } \\
\text { indications during a dwell state may be } \\
\text { reported as green or inactive. (common to } \\
\text { mid-block signals) }\end{array}$ \\
\hline 64 & $\begin{array}{l}\text { Overlap Begin Red } \\
\text { Clearance }\end{array}$ & $\begin{array}{l}\text { Overlap \# (as number } \\
A=1 B=2 \text {, etc) }\end{array}$ & $\begin{array}{l}\text { Set when overlap begins timing red } \\
\text { clearance intervals. }\end{array}$ \\
\hline 65 & $\begin{array}{l}\text { Overlap Off (Inactive with } \\
\text { red indication) }\end{array}$ & $\begin{array}{l}\text { Overlap \# (as number } \\
A=1 B=2 \text {, etc) }\end{array}$ & $\begin{array}{l}\text { Set when overlap has completed all timing, } \\
\text { allowing any conflicting phase next to begin } \\
\text { service. }\end{array}$ \\
\hline 66 & Overlap Dark & $\begin{array}{l}\text { Overlap \# (as number } \\
A=1 B=2 \text {, etc) }\end{array}$ & $\begin{array}{l}\text { Set when overlap head is set dark (no active } \\
\text { outputs). The end of this interval shall be } \\
\text { recorded by either an overlap off state or } \\
\text { other active overlap state. }\end{array}$ \\
\hline 67 & $\begin{array}{l}\text { Pedestrian Overlap Begin } \\
\text { Walk }\end{array}$ & $\begin{array}{l}\text { Overlap \# (as number } \\
A=1 B=2 \text {, etc) }\end{array}$ & Set when walk indication becomes active. \\
\hline 68 & $\begin{array}{l}\text { Pedestrian Overlap Begin } \\
\text { Clearance }\end{array}$ & $\begin{array}{l}\text { Overlap \# (as number } \\
A=1 B=2 \text {, etc) }\end{array}$ & $\begin{array}{l}\text { Set when flashing don't walk indication } \\
\text { becomes active. }\end{array}$ \\
\hline 69 & $\begin{array}{l}\text { Pedestrian Overlap Begin } \\
\text { Solid Don't Walk }\end{array}$ & $\begin{array}{l}\text { Overlap \# (as number } \\
A=1 B=2 \text {, etc) }\end{array}$ & $\begin{array}{l}\text { Set when don't walk indication becomes } \\
\text { solid (non flashing) from either termination of } \\
\text { ped clearance, or head illumination after a } \\
\text { ped dark interval. }\end{array}$ \\
\hline 70 & Pedestrian Overlap Dark & $\begin{array}{l}\text { Overlap \# (as number } \\
A=1 B=2 \text {, etc) }\end{array}$ & Set when the pedestrian outputs are set off. \\
\hline 71 & $\begin{array}{l}\text { Advance Warning Sign } \\
\text { On }\end{array}$ & $\begin{array}{l}\text { Overlap \# (as number } \\
A=1 B=2 \text {, etc) }\end{array}$ & $\begin{array}{l}\text { Set when advance warning sign becomes } \\
\text { active. }\end{array}$ \\
\hline 72 & $\begin{array}{l}\text { Advance Warning Sign } \\
\text { Off }\end{array}$ & $\begin{array}{l}\text { Overlap \# (as number } \\
A=1 B=2 \text {, etc) }\end{array}$ & $\begin{array}{l}\text { Set when advance warning sign becomes } \\
\text { inactive. }\end{array}$ \\
\hline $73-80$ & $\begin{array}{l}\text { Overlap events reserved } \\
\text { for future use. }\end{array}$ & & \\
\hline
\end{tabular}




\begin{tabular}{|c|c|c|c|}
\hline \multicolumn{4}{|c|}{ Detector Events: } \\
\hline 81 & Detector Off & DET Channel \# & $\begin{array}{l}\text { Detector on and off events shall be triggered } \\
\text { post any detector delay/extension } \\
\text { processing. }\end{array}$ \\
\hline 82 & Detector On & DET Channel \# & $\begin{array}{l}\text { Detector on and off events shall be triggered } \\
\text { post any detector delay/extension } \\
\text { processing. }\end{array}$ \\
\hline 83 & Detector Restored & DET Channel \# & $\begin{array}{l}\text { Detector restored to non-failed state by } \\
\text { either manual restoration or re-enabling via } \\
\text { continued diagnostics. }\end{array}$ \\
\hline 84 & Detector Fault- Other & DET Channel \# & $\begin{array}{l}\text { Detector failure logged upon local controller } \\
\text { diagnostics only (not system diagnostics). }\end{array}$ \\
\hline 85 & $\begin{array}{l}\text { Detector Fault- Watchdog } \\
\text { Fault }\end{array}$ & DET Channel \# & $\begin{array}{l}\text { Detector failure logged upon local controller } \\
\text { diagnostics only (not system diagnostics). }\end{array}$ \\
\hline 86 & $\begin{array}{l}\text { Detector Fault- Open } \\
\text { Loop Fault }\end{array}$ & DET Channel \# & $\begin{array}{l}\text { Detector failure logged upon local controller } \\
\text { diagnostics only (not system diagnostics). }\end{array}$ \\
\hline 87 & $\begin{array}{l}\text { Detector Fault- Shorted } \\
\text { Loop Fault }\end{array}$ & DET Channel \# & $\begin{array}{l}\text { Detector failure logged upon local controller } \\
\text { diagnostics only (not system diagnostics). }\end{array}$ \\
\hline 88 & $\begin{array}{l}\text { Detector Fault- Excessive } \\
\text { Change Fault }\end{array}$ & DET Channel \# & $\begin{array}{l}\text { Detector failure logged upon local controller } \\
\text { diagnostics only (not system diagnostics). }\end{array}$ \\
\hline 89 & PedDetector Off & DET Channel \# & $\begin{array}{l}\text { Ped detector events shall be triggered post } \\
\text { any detector delay/extension processing } \\
\text { and may be set multiple times for a single } \\
\text { pedestrian call. (with future intent to } \\
\text { eventually support ped presence and } \\
\text { volume). }\end{array}$ \\
\hline 90 & PedDetector On & DET Channel \# & $\begin{array}{l}\text { Ped detector events shall be triggered post } \\
\text { any detector delay/extension processing } \\
\text { and may be set multiple times for a single } \\
\text { pedestrian call. (with future intent to } \\
\text { eventually support ped presence and } \\
\text { volume). }\end{array}$ \\
\hline 91 & $\begin{array}{l}\text { Pedestrian Detector } \\
\text { Failed }\end{array}$ & Ped Det \# (1-255) & $\begin{array}{l}\text { Detector failure logged upon local controller } \\
\text { diagnostics only (not system diagnostics). }\end{array}$ \\
\hline 92 & $\begin{array}{l}\text { Pedestrian Detector } \\
\text { Restored }\end{array}$ & Ped Det \# (1-255) & $\begin{array}{l}\text { Detector failure logged upon local controller } \\
\text { diagnostics only (not system diagnostics). }\end{array}$ \\
\hline 93 & TSP Detector Off & TSP \#(1-255) & $\begin{array}{l}\text { TSP detector events shall be triggered post } \\
\text { any detector delay/extension processing. }\end{array}$ \\
\hline 94 & TSP Detector On & TSP \#(1-255) & $\begin{array}{l}\text { TSP detector events shall be triggered post } \\
\text { any detector delay/extension processing. }\end{array}$ \\
\hline $95-100$ & $\begin{array}{l}\text { Detector events reserved } \\
\text { for future use. }\end{array}$ & & \\
\hline
\end{tabular}




\begin{tabular}{|c|c|c|c|}
\hline \multicolumn{4}{|c|}{ Preemption Events: } \\
\hline 101 & $\begin{array}{l}\text { Preempt Advance } \\
\text { Warning Input }\end{array}$ & Preempt \# (1-255) & $\begin{array}{l}\text { Set when preemption advance warning input } \\
\text { is activated. }\end{array}$ \\
\hline 102 & Preempt (Call) Input On & Preempt \# (1-255) & $\begin{array}{l}\text { Set when preemption input is activated. } \\
\text { (prior to preemption delay timing) May be } \\
\text { set multiple times if input is intermittent } \\
\text { during preemption service. }\end{array}$ \\
\hline 103 & $\begin{array}{l}\text { Preempt Gate Down Input } \\
\text { Received }\end{array}$ & Preempt \# (1-255) & $\begin{array}{l}\text { Set when gate down input is received by the } \\
\text { controller (if available). }\end{array}$ \\
\hline 104 & Preempt (Call) Input Off & Preempt \# (1-255) & $\begin{array}{l}\text { Set when preemption input is de-activated. } \\
\text { May be set multiple times if input is } \\
\text { intermittent preemption service. }\end{array}$ \\
\hline 105 & Preempt Entry Started & Preempt \# (1-255) & $\begin{array}{l}\text { Set when preemption delay expires, and } \\
\text { controller begins transition timing (force off) } \\
\text { to serve preemption. }\end{array}$ \\
\hline 106 & $\begin{array}{l}\text { Preemption Begin Track } \\
\text { Clearance }\end{array}$ & Preempt \# (1-255) & $\begin{array}{l}\text { Set when track clearance phases are green } \\
\text { and track clearance timing begins. }\end{array}$ \\
\hline 107 & $\begin{array}{l}\text { Preemption Begin Dwell } \\
\text { Service }\end{array}$ & Preempt \# (1-255) & $\begin{array}{l}\text { Set when preemption dwell or limited } \\
\text { service begins, or minimum dwell timer is } \\
\text { reset due to call drop and reapplication. }\end{array}$ \\
\hline 108 & $\begin{array}{l}\text { Preemption Link Active } \\
\text { On }\end{array}$ & Preempt \# (1-255) & $\begin{array}{l}\text { Set when linked preemptor input is applied } \\
\text { from active preemptor. }\end{array}$ \\
\hline 109 & $\begin{array}{l}\text { Preemption Link Active } \\
\text { Off }\end{array}$ & Preempt \# (1-255) & $\begin{array}{l}\text { Set when linked preemptor input is dropped } \\
\text { from active preemptor. }\end{array}$ \\
\hline 110 & $\begin{array}{l}\text { Preemption Max } \\
\text { Presence Exceeded }\end{array}$ & Preempt \# (1-255) & $\begin{array}{l}\text { Set when preemption max presence timer is } \\
\text { exceeded, and preemption input is released } \\
\text { from service. }\end{array}$ \\
\hline 111 & $\begin{array}{l}\text { Preemption Begin Exit } \\
\text { Interval }\end{array}$ & Preempt \# (1-255) & $\begin{array}{l}\text { Set when preemption exit interval phases } \\
\text { are green and exit timing begins. }\end{array}$ \\
\hline 112 & TSP Check In & TSP \#(1-255) & Set when request for priority is received. \\
\hline 113 & $\begin{array}{l}\text { TSP Adjustment to Early } \\
\text { Green }\end{array}$ & TSP \#(1-255) & $\begin{array}{l}\text { Set when controller is adjusting active cycle } \\
\text { to accommodate early service to TSP } \\
\text { phases. }\end{array}$ \\
\hline 114 & $\begin{array}{l}\text { TSP Adjustment to } \\
\text { Extend Green }\end{array}$ & TSP \#(1-255) & $\begin{array}{l}\text { Set when controller is adjusting active cycle } \\
\text { to accommodate extended service to TSP } \\
\text { phases. }\end{array}$ \\
\hline 115 & TSP Check Out & TSP \#(1-255) & Set when request for priority is retracted. \\
\hline 116 & Preemption Force Off & Preempt \# (1-255) & $\begin{array}{l}\text { Set when preemption force off is applied to } \\
\text { the active cycle. }\end{array}$ \\
\hline 117 & TSP Early Force Off & TSP \#(1-255) & $\begin{array}{l}\text { Set when TSP early force off is applied to } \\
\text { the active cycle. }\end{array}$ \\
\hline 118 & TSP Service Start & TSP \#(1-255) & Set when requested TSP service begins. \\
\hline 119 & TSP Service End & TSP \#(1-255) & Set when requested TSP service ends. \\
\hline
\end{tabular}


120-130 Preemption/TSP Events reserved for future use 


\begin{tabular}{|c|c|c|c|}
\hline \multicolumn{4}{|c|}{ Coordination Events: } \\
\hline 131 & Coord Pattern Change & Pattern \# (0-255) & $\begin{array}{l}\text { Coordination pattern that is actively running } \\
\text { in the controller. (Highest priority of TOD, } \\
\text { System or manual command). This event } \\
\text { will not be reapplied if coordination is } \\
\text { temporarily suspended for preemption or } \\
\text { other external control. }\end{array}$ \\
\hline 132 & Cycle Length Change & Seconds (0-255) & $\begin{array}{l}\text { This event shall be populated upon selection } \\
\text { of a new coordination pattern change that } \\
\text { selects a new cycle length. Cycle lengths in } \\
\text { excess of } 255 \text { shall record this event with a } \\
255 \text { parameter, along with event code } 156 \text {. }\end{array}$ \\
\hline 133 & Offset Length Change & Seconds (0-255) & $\begin{array}{l}\text { This event shall be populated upon selection } \\
\text { of a new coordination pattern change that } \\
\text { selects a new cycle length. Offsets in } \\
\text { excess of } 255 \text { shall record this event with a } \\
255 \text { parameter, requiring controller database } \\
\text { lookup for this actual value. }\end{array}$ \\
\hline 134 & Split 1 Change & $\begin{array}{l}\text { New Split Time in } \\
\text { Seconds }(0-255)\end{array}$ & $\begin{array}{l}\text { Split change events shall be populated upon } \\
\text { selection of a new coordination pattern as } \\
\text { well as during a split change to an active } \\
\text { pattern via Signal Control and Prioritization } \\
\text { (SCP), Adaptive Control System (ACS) Lite } \\
\text { or other adaptive control system. }\end{array}$ \\
\hline 135 & Split 2 Change & $\begin{array}{l}\text { New Split Time in } \\
\text { Seconds }(0-255)\end{array}$ & \\
\hline 136 & Split 3 Change & $\begin{array}{l}\text { New Split Time in } \\
\text { Seconds }(0-255)\end{array}$ & \\
\hline 137 & Split 4 Change & $\begin{array}{l}\text { New Split Time in } \\
\text { Seconds }(0-255)\end{array}$ & \\
\hline 138 & Split 5 Change & $\begin{array}{l}\text { New Split Time in } \\
\text { Seconds }(0-255)\end{array}$ & \\
\hline 139 & Split 6 Change & $\begin{array}{l}\text { New Split Time in } \\
\text { Seconds }(0-255)\end{array}$ & \\
\hline 140 & Split 7 Change & $\begin{array}{l}\text { New Split Time in } \\
\text { Seconds }(0-255)\end{array}$ & \\
\hline 141 & Split 8 Change & $\begin{array}{l}\text { New Split Time in } \\
\text { Seconds }(0-255)\end{array}$ & \\
\hline 142 & Split 9 Change & $\begin{array}{l}\text { New Split Time in } \\
\text { Seconds }(0-255)\end{array}$ & \\
\hline 143 & Split 10 Change & $\begin{array}{l}\text { New Split Time in } \\
\text { Seconds }(0-255)\end{array}$ & \\
\hline 144 & Split 11 Change & $\begin{array}{l}\text { New Split Time in } \\
\text { Seconds }(0-255)\end{array}$ & \\
\hline
\end{tabular}




\begin{tabular}{|c|c|c|c|}
\hline 145 & Split 12 Change & $\begin{array}{l}\text { New Split Time in } \\
\text { Seconds }(0-255)\end{array}$ & \\
\hline 146 & Split 13 Change & $\begin{array}{l}\text { New Split Time in } \\
\text { Seconds }(0-255)\end{array}$ & \\
\hline 147 & Split 14 Change & $\begin{array}{l}\text { New Split Time in } \\
\text { Seconds }(0-255)\end{array}$ & \\
\hline 148 & Split 15 Change & $\begin{array}{l}\text { New Split Time in } \\
\text { Seconds }(0-255)\end{array}$ & \\
\hline 149 & Split 16 Change & $\begin{array}{l}\text { New Split Time in } \\
\text { Seconds }(0-255)\end{array}$ & \\
\hline 150 & Coord cycle state change & $\begin{array}{l}\text { Parameter }(0-6) \\
\text { defined as: } \\
0=\text { Free } \\
1=\text { In Step } \\
2=\text { Transition - Add } \\
3=\text { Transition - } \\
\text { Subtract } \\
4=\text { Transition - Dwell } \\
5=\text { Local Zero } \\
6=\text { Begin Pickup } \\
7=\text { Master Cycle } \\
\text { Zero }\end{array}$ & \\
\hline 151 & $\begin{array}{l}\text { Coordinated phase yield } \\
\text { point }\end{array}$ & Phase \# (1-255) & $\begin{array}{l}\text { Set once per cycle for each coordinated } \\
\text { phase when controller suspends the } \\
\text { coordinated phase. }\end{array}$ \\
\hline 152 & Coordinated phase begin & Phase \# (1-255) & Set when coordinated phase begins. \\
\hline 153 & Logic Statement True & $\begin{array}{l}\text { Logic Statement \# (1- } \\
255)\end{array}$ & $\begin{array}{l}\text { Set when the logic statement evaluation } \\
\text { changes from "False" to "True". }\end{array}$ \\
\hline 154 & Logic Statement False & $\begin{array}{l}\text { Logic Statement \# (1- } \\
255)\end{array}$ & $\begin{array}{l}\text { Set when the logic statement evaluation } \\
\text { changes from "True" to "False". }\end{array}$ \\
\hline 155 & $\begin{array}{l}\text { Unit Control Status } \\
\text { Change }\end{array}$ & $\begin{array}{l}\text { Parameter }(1-10) \\
\text { defined as: } \\
1=\text { Other } \\
2 \text { = System Control } \\
3 \text { = System Standby } \\
4=\text { Backup Mode } \\
5=\text { Manual } \\
6=\text { Timebase } \\
7=\text { Interconnect } \\
8=\text { Interconnect } \\
\text { Backup } \\
9=\text { Remote Manual } \\
\text { Control } \\
10=\text { Local Manual } \\
\text { Control }\end{array}$ & See NTCIP 1202v0326 5.4 .5 for definition. \\
\hline
\end{tabular}




\begin{tabular}{|l|l|l|l|}
\hline 156 & $\begin{array}{l}\text { Additional Cycle Length } \\
\text { Change }\end{array}$ & Seconds (0-255) & $\begin{array}{l}\text { Set simultaneously with Event Code 132 } \\
\text { when existing cycle length exceeds 255 } \\
\text { seconds. When this Event Code 156 is } \\
\text { used, Event Code 132 shall be 255, where } \\
\text { the value of this Event Code 156 will be the } \\
\text { additional cycle length above 255 (in } \\
\text { seconds). Cycle lengths longer than 510 } \\
\text { seconds will require a controller look up. }\end{array}$ \\
\hline $157-170$ & $\begin{array}{l}\text { Coordination events } \\
\text { reserved for future use. }\end{array}$ & \\
\hline
\end{tabular}




\begin{tabular}{|c|c|c|c|}
\hline \multicolumn{4}{|c|}{ Cabinet / System Events: } \\
\hline 171 & Test Input On & $\begin{array}{l}\text { Test Input \# (as } \\
\text { number } A=1 B=2 \text {, } \\
\text { etc) }\end{array}$ & $\begin{array}{l}\text { Cabinet test or special function input as } \\
\text { defined by the local controller. }\end{array}$ \\
\hline 172 & Test Input Off & $\begin{array}{l}\text { Test Input \# (as } \\
\text { number } A=1 \quad B=2 \text {, } \\
\text { etc) }\end{array}$ & $\begin{array}{l}\text { Cabinet test or special function input as } \\
\text { defined by the local controller. }\end{array}$ \\
\hline 173 & Unit Flash Status Change & $\begin{array}{l}\text { NTCIP Flash state \# } \\
(0-255)\end{array}$ & See NTCIP 1202 2.4.6 for definition. \\
\hline 174 & $\begin{array}{l}\text { Unit Alarm Status } 1 \\
\text { Change }\end{array}$ & $\begin{array}{l}\text { NTCIP Alarm Status } \\
1 \#(0-255)\end{array}$ & See NTCIP 1202 2.4.8 for definition. \\
\hline 175 & $\begin{array}{l}\text { Alarm Group State } \\
\text { Change }\end{array}$ & $\begin{array}{l}\text { NTCIP Alarm Group } \\
\text { State }(0-255)\end{array}$ & See NTCIP 1202 2.4.12.2 for definition. \\
\hline 176 & $\begin{array}{l}\text { Special Function Output } \\
\text { On }\end{array}$ & $\begin{array}{l}\text { Special Function \# } \\
(0-255)\end{array}$ & $\begin{array}{l}\text { Special function output as defined by the } \\
\text { local controller. }\end{array}$ \\
\hline 177 & $\begin{array}{l}\text { Special Function Output } \\
\text { Off }\end{array}$ & $\begin{array}{l}\text { Special Function \# } \\
(0-255)\end{array}$ & $\begin{array}{l}\text { Special function output as defined by the } \\
\text { local controller. }\end{array}$ \\
\hline 178 & $\begin{array}{l}\text { Manual control enable } \\
\text { On/Off }\end{array}$ & $\begin{array}{l}\text { Manual control } \\
\text { enable On/Off \# }(1,0)\end{array}$ & $\begin{array}{l}\text { Special function output as defined by the } \\
\text { local controller. }\end{array}$ \\
\hline 179 & Interval Advance On/Off & $\begin{array}{l}\text { Interval Advance } \\
\text { On/Off \# }(1,0)\end{array}$ & $\begin{array}{l}\text { Leading edge on (1), lagging edge }(0) \\
\text { optional. }\end{array}$ \\
\hline 180 & Stop Time Input On/Off & $\begin{array}{l}\text { Stop Time Input } \\
\text { Advance On/Off \# } \\
(1,0)\end{array}$ & $\begin{array}{l}\text { Set when stop time input is applied or } \\
\text { removed, regardless of source of stop or } \\
\text { state. }\end{array}$ \\
\hline 181 & Controller Clock Updated & $\begin{array}{l}\text { Optional parameter: } \\
\text { Time correction in } \\
\text { Seconds }(0-255)\end{array}$ & $\begin{array}{l}\text { Set when the controller OS clock is adjusted } \\
\text { via communications, OS command, or } \\
\text { external input. }\end{array}$ \\
\hline 182 & Power Failure Detected & True (1) & $\begin{array}{l}\text { Line voltage drops between } 0-89 \text { volts } A C \\
\text { for more than } 100 \mathrm{~ms} \text {. }\end{array}$ \\
\hline 184 & Power Restored & True (1) & $\begin{array}{l}\text { Line voltage applied/reapplied greater than } \\
98 \text { volts AC. }\end{array}$ \\
\hline 185 & Vendor Specific Alarm & $\begin{array}{l}\text { Vendor defined } \\
\text { parameter }\end{array}$ & $\begin{array}{l}\text { Placeholder for generic failure/alarm types } \\
\text { as defined by vendor. }\end{array}$ \\
\hline $186-199$ & $\begin{array}{l}\text { Cabinet/System events } \\
\text { reserved for future use. }\end{array}$ & & \\
\hline 200 & Alarm On & $\begin{array}{l}\text { Alarm \# (as number } \\
A=1 B=2 \text {, etc) }\end{array}$ & Set when cabinet/system alarm is activated. \\
\hline 201 & Alarm Off & $\begin{array}{l}\text { Alarm \# (as number } \\
A=1 B=2 \text {, etc) }\end{array}$ & Set when cabinet/system alarm is released. \\
\hline 202 & Aux Switch On/Off & $\begin{array}{l}\text { Aux switch On/Off \# } \\
(1,0)\end{array}$ & $\begin{array}{l}\text { Set when local controller aux switch is active } \\
\text { (1) or inactive (0) }\end{array}$ \\
\hline
\end{tabular}




\begin{tabular}{|c|c|c|c|}
\hline 203 & Split 17 Change & $\begin{array}{l}\text { New Split Time in } \\
\text { Seconds }(0-255)\end{array}$ & $\begin{array}{l}\text { Split change events shall be populated upon } \\
\text { selection of a new coordination pattern as } \\
\text { well as during a split change to an active } \\
\text { pattern via Signal Control and Prioritization } \\
\text { (SCP), Adaptive Control System (ACS) Lite } \\
\text { or other adaptive control system. }\end{array}$ \\
\hline 204 & Split 18 Change & $\begin{array}{l}\text { New Split Time in } \\
\text { Seconds }(0-255)\end{array}$ & \\
\hline 205 & Split 19 Change & $\begin{array}{l}\text { New Split Time in } \\
\text { Seconds }(0-255)\end{array}$ & \\
\hline 206 & Split 20 Change & $\begin{array}{l}\text { New Split Time in } \\
\text { Seconds }(0-255)\end{array}$ & \\
\hline 207 & Split 21 Change & $\begin{array}{l}\text { New Split Time in } \\
\text { Seconds }(0-255)\end{array}$ & \\
\hline 208 & Split 22 Change & $\begin{array}{l}\text { New Split Time in } \\
\text { Seconds }(0-255)\end{array}$ & \\
\hline 209 & Split 23 Change & $\begin{array}{l}\text { New Split Time in } \\
\text { Seconds }(0-255)\end{array}$ & \\
\hline 210 & Split 24 Change & $\begin{array}{l}\text { New Split Time in } \\
\text { Seconds }(0-255)\end{array}$ & \\
\hline 211 & Split 25 Change & $\begin{array}{l}\text { New Split Time in } \\
\text { Seconds }(0-255)\end{array}$ & \\
\hline 212 & Split 26 Change & $\begin{array}{l}\text { New Split Time in } \\
\text { Seconds }(0-255)\end{array}$ & \\
\hline 213 & Split 27 Change & $\begin{array}{l}\text { New Split Time in } \\
\text { Seconds }(0-255)\end{array}$ & \\
\hline 214 & Split 28 Change & $\begin{array}{l}\text { New Split Time in } \\
\text { Seconds }(0-255)\end{array}$ & \\
\hline 215 & Split 29 Change & $\begin{array}{l}\text { New Split Time in } \\
\text { Seconds }(0-255)\end{array}$ & \\
\hline 216 & Split 30 Change & $\begin{array}{l}\text { New Split Time in } \\
\text { Seconds }(0-255)\end{array}$ & \\
\hline 217 & Split 31 Change & $\begin{array}{l}\text { New Split Time in } \\
\text { Seconds }(0-255)\end{array}$ & \\
\hline 218 & Split 32 Change & $\begin{array}{l}\text { New Split Time in } \\
\text { Seconds }(0-255)\end{array}$ & \\
\hline $219-255$ & Reserved for future use & & \\
\hline
\end{tabular}




\section{Appendix}

\section{Executive Summary}

The first version of the Indiana Traffic Signal Hi Resolution Data Logger Enumerations was published in 2012 and has been widely adopted by the traffic industry. Since the initial specification was released, traffic signal controller manufacturers, including Econolite, Siemens, Peek, McCain, Intelight, and Trafficware, have incorporated the standardized data logging enumerations into their controllers on a software level. As the enumerations have been adopted and implemented, small discrepancies in measure calculations and ambiguities in enumeration definitions have emerged which has resulted in varying implementation between manufacturers.

To address the current discrepancies, Pooled Fund Project TPF-5(377) was created to enhance the definitions and develop new enumerations where appropriate. The aim of the project is to enhance and expand the Traffic Signal Performance Measures that were developed under the original Pooled Fund Project TPF-5(258). The work to enhance the enumerations involved identifying as many issues as possible with the current 2012 specification, modify existing definitions as needed, and incorporate new enumerations to address all concerns and issues.

In March of 2019, a roundtable meeting was held at Purdue University, West Lafayette, IN where representatives from FHWA, state Departments of Transportation, private sectors, and other stakeholders participated. The University of Alabama team presented potential inconsistencies and ambiguities in the current data logger specifications and proposed relevant solutions regarding the issues. Issues from each state were discussed, and each controller manufacturer presented their notes and thoughts. After the panel meeting, The University of Alabama team reached out to all manufacturers to collect additional thoughts and suggestions for modifications to the specification. More than thirty issues and suggestions were collected, considered, and assembled in this updated document.

This second version of the specification has addresses all known discrepancies and issues with the 2012 data logger enumerations and presents solutions relevant to the issues in the form of modified definitions. In addition, this report introduces several new enumerations and descriptions which were gathered from participating agencies and vendors. The Appendix section of this report discusses all the changes made on the updated hi resolution traffic data logger enumerations. One final comment from manufacturers that was noted is the need for testing of the enumerations. A standard protocol of software testing should be used to verify that the enumerations are consistent from one manufacturer to another. 


\section{New Event Code 13}

\begin{tabular}{|l|l|l|l|}
\hline Event Code & Event Descriptor & Parameter & Description \\
\hline 13 & $\begin{array}{l}\text { Extension Timer } \\
\text { Gap Out }\end{array}$ & Phase \# (1-255) & $\begin{array}{l}\text { Set when extension timer gaps } \\
\text { out. }\end{array}$ \\
\hline
\end{tabular}

Note: Event Code (EC) 13 is proposed to eliminate the ambiguity in EC 4 (Phase Gap Out) definition. Different controller manufacturers report EC 4 differently (i.e., some controllers report EC 4 multiple times during single green and some report once at the end of the green). The new EC 13 is set when extension timer gaps out.

\section{New Event Code 14}

\begin{tabular}{|l|l|l|l|}
\hline Event Code & Event Descriptor & Parameter & Description \\
\hline 14 & Phase Skipped & Phase \# (1-255) & $\begin{array}{l}\text { Set when phase in the } \\
\text { programmed ring is skipped for } \\
\text { any reason. }\end{array}$ \\
\hline
\end{tabular}

Note: Event Code (EC) 14 is proposed to help verify if any phase in the programmed ring is skipped for any reason. This event needs to be logged before the start of green of the next phase served. This event code was proposed by Dan Nelson (Siemens Industry, Inc.).

\section{New Event Code 15}

\begin{tabular}{|l|l|l|l|}
\hline Event Code & Event Descriptor & Parameter & Description \\
\hline 15 & $\begin{array}{l}\text { Extension Timer } \\
\text { Reduction Start }\end{array}$ & Phase \# (1-255) & $\begin{array}{l}\text { Set when extension timer starts } \\
\text { to reduce (the time before } \\
\text { reduction). }\end{array}$ \\
\hline
\end{tabular}

Note: Event Code (EC) 15 is proposed to log the event when extension timer begins to reduce after the arrival of a conflicting call and before the time to reduce. This event code was proposed by Dan Nelson (Siemens Industry, Inc.). 


\section{New Event Code 16}

\begin{tabular}{|l|l|l|l|}
\hline Event Code & Event Descriptor & Parameter & Description \\
\hline 16 & $\begin{array}{l}\text { Extension Timer } \\
\text { Minimum } \\
\text { Achieved }\end{array}$ & Phase \# (1-255) & $\begin{array}{l}\text { Set when extension timer } \\
\text { minimum is reached (after the } \\
\text { time to reduce). }\end{array}$ \\
\hline
\end{tabular}

Note: Event Code (EC) 16 is proposed to capture the event when extension timer minimum is achieved upon the expiration of time to reduce. This event code was proposed by Dan Nelson (Siemens Industry, Inc.).

New Event Code 17

\begin{tabular}{|l|l|l|l|}
\hline Event Code & Event Descriptor & Parameter & Description \\
\hline 17 & $\begin{array}{l}\text { Added Initial } \\
\text { Complete }\end{array}$ & Phase \# (1-255) & $\begin{array}{l}\text { Set when phase added initial } \\
\text { timer expires. }\end{array}$ \\
\hline
\end{tabular}

Note: Event Code (EC) 17 is proposed to log the controller event when phase added initial timer expires. This event code was proposed by Dan Nelson (Siemens Industry, Inc.).

\section{New Event Code 18}

\begin{tabular}{|l|l|l|l|}
\hline Event Code & Event Descriptor & Parameter & Description \\
\hline 18 & $\begin{array}{l}\text { Next Phase } \\
\text { Decision }\end{array}$ & Phase \# (1-255) & $\begin{array}{l}\text { Set when the controller } \\
\text { determines a phase will be next } \\
\text { to begin green after the current } \\
\text { active phase(s) end red } \\
\text { clearance. }\end{array}$ \\
\hline
\end{tabular}

Note: Event Code (EC) 18 is proposed to log the event when controller determines a phase will be next to begin green after the current active phase ends red clearance. This event code was proposed by Howell Li (Purdue University.). 


\section{New Event Code 19}

\begin{tabular}{|l|l|l|l|}
\hline Event Code & Event Descriptor & Parameter & Description \\
\hline 19 & TSP Early Force Off & Phase \# (1-255) & $\begin{array}{l}\text { Set when TSP early force off is } \\
\text { applied to an active phase. }\end{array}$ \\
\hline
\end{tabular}

Note: Event Code (EC) 19 is proposed to log the event when controller applies early force off to an active phase during Transit Signal Priority (TSP) operation. This event code was proposed by Mark Taylor (UDOT).

New Event Code 20

\begin{tabular}{|l|l|l|l|}
\hline Event Code & Event Descriptor & Parameter & Description \\
\hline 20 & $\begin{array}{l}\text { Preemption Force } \\
\text { Off }\end{array}$ & Phase \# (1-255) & $\begin{array}{l}\text { Set when controller applies } \\
\text { preemption force off to the } \\
\text { active cycle. }\end{array}$ \\
\hline
\end{tabular}

Note: Event Code (EC) 20 is proposed to log the event when controller applies preemption force off to the active cycle. This event code was proposed by Howell Li (Purdue University).

\section{New Event Code 25}

\begin{tabular}{|l|l|l|l|}
\hline Event Code & Event Descriptor & Parameter & Description \\
\hline 25 & $\begin{array}{l}\text { Extended } \\
\text { Pedestrian Change } \\
\text { Interval }\end{array}$ & Phase \# (1-255) & $\begin{array}{l}\text { Set when extended pedestrian } \\
\text { change interval is requested by } \\
\text { pressing the pedestrian push } \\
\text { button for two (2) seconds. See } \\
\text { 2009 MUTCD Section 4E.13 - } \\
\text { Accessible Pedestrian Signals and } \\
\text { Detectors - Extended Pushbutton } \\
\text { Press Features for more details. }\end{array}$ \\
\hline
\end{tabular}

Note: Event Code (EC) 25 is proposed to log the event when extended pedestrian change interval is requested by pressing the pedestrian push button for two (2) seconds. This event code was proposed by Dan Nelson (Siemens Industry, Inc.). 


\section{New Event Code 26}

\begin{tabular}{|l|l|l|l|}
\hline Event Code & Event Descriptor & Parameter & Description \\
\hline 26 & $\begin{array}{l}\text { Oversized } \\
\text { Pedestrian Served }\end{array}$ & Phase \# (1-255) & $\begin{array}{l}\text { Set when pedestrian phase is } \\
\text { active beyond pedestrian change } \\
\text { interval or force off point. }\end{array}$ \\
\hline
\end{tabular}

Note: Event Code (EC) 26 is proposed to log the event when pedestrian phase is still active beyond pedestrian change interval or force off point due to heavy pedestrian demand. This event code was proposed by Tom Stiles (Intelight Inc.).

New Event Code 50 and 51

\begin{tabular}{|l|l|l|l|}
\hline Event Code & Event Descriptor & Parameter & Description \\
\hline 50 & MAX 1 In-Effect & Phase \# (1-255) & $\begin{array}{l}\text { Set when maximum green (MAX } \\
\text { 1) interval is in-effect for the } \\
\text { active phase. }\end{array}$ \\
\hline 51 & MAX 2 In-Effect & Phase \# (1-255) & $\begin{array}{l}\text { Set when maximum green (MAX } \\
\text { 2) interval is in-effect for the } \\
\text { active phase. }\end{array}$ \\
\hline
\end{tabular}

Note: Event Codes (EC) 50 and 51 are proposed to log the events when controller maximum green (MAX 1 or MAX 2) interval is in-effect for the active phase. These event codes were proposed by Steve Gault (PennDOT) and Matthew Carlisle (NCDOT).

New Event Code 52

\begin{tabular}{|l|l|l|l|}
\hline Event Code & Event Descriptor & Parameter & Description \\
\hline 52 & $\begin{array}{l}\text { Dynamic MAX In- } \\
\text { Effect }\end{array}$ & Phase \# (1-255) & $\begin{array}{l}\text { Set when dynamic max interval is } \\
\text { in-effect for the active phase. } \\
\text { This event shall be populated } \\
\text { upon termination of MAX green } \\
\text { (MAX 1 or MAX 2) interval. }\end{array}$ \\
\hline
\end{tabular}

Note: Event Code (EC) 52 is proposed to log the event dynamic max interval is in-effect for the active phase. This event shall be populated upon termination of MAX green (MAX 1 or MAX 2) interval. This event code was proposed by Steve Gault (PennDOT). 
New Event Code 53 and 54

\begin{tabular}{|l|l|l|l|}
\hline Event Code & Event Descriptor & Parameter & Description \\
\hline 53 & $\begin{array}{l}\text { Dynamic MAX Step } \\
\text { Up }\end{array}$ & Phase \# (1-255) & $\begin{array}{l}\text { Set when dynamic max interval } \\
\text { steps up for the active phase } \\
\text { (initially after two consecutive } \\
\text { phase max out events). }\end{array}$ \\
\hline 54 & $\begin{array}{l}\text { Dynamic MAX Step } \\
\text { Down }\end{array}$ & Phase \# (1-255) & $\begin{array}{l}\text { Set when dynamic max interval } \\
\text { steps down for the active phase } \\
\text { (initially after two consecutive } \\
\text { phase gap out events). }\end{array}$ \\
\hline
\end{tabular}

Note: Event Code (EC) 53 is proposed to log the event dynamic max interval steps up for the active phase (initially after two consecutive phase max out events). Dynamic max step value will be added to the running max or adaptive maximum until the running max or adaptive maximum is greater than the larger of the normal maximum or dynamic maximum. Event Code (EC) 54 is proposed to log the event when dynamic max interval steps down for the active phase (initially after two consecutive phase gap out events). Dynamic max step value will be subtracted from the running max or adaptive maximum until the running max or adaptive maximum is less than the smaller of the normal maximum or dynamic maximum. These event codes were proposed by Steve Gault (PennDOT) and Matthew Carlisle (NCDOT). 
New Event Code 55 and 56

\begin{tabular}{|l|l|l|l|}
\hline Event Code & Event Descriptor & Parameter & Description \\
\hline 55 & $\begin{array}{l}\text { Advance Warning } \\
\text { Sign On }\end{array}$ & Phase \# (1-255) & $\begin{array}{l}\text { Set when advance warning sign is } \\
\text { On. }\end{array}$ \\
\hline 56 & $\begin{array}{l}\text { Advance Warning } \\
\text { Sign Off }\end{array}$ & Phase \# (1-255) & $\begin{array}{l}\text { Set when advance warning sign is } \\
\text { Off. }\end{array}$ \\
\hline
\end{tabular}

Note: Event Code (EC) 55 is proposed to log the event when advance warning sign is on. Event Code (EC) 56 is proposed to log the event when advance warning sign is off. These event codes were proposed by Dan Nelson (Siemens Industry, Inc.).

New Event Code 71 and 72

\begin{tabular}{|l|l|l|l|}
\hline Event Code & Event Descriptor & Parameter & Description \\
\hline 71 & $\begin{array}{l}\text { Advance Warning } \\
\text { Sign On }\end{array}$ & $\begin{array}{l}\text { Overlap \# (as } \\
\text { number A=1 B=2, } \\
\text { etc) }\end{array}$ & $\begin{array}{l}\text { Set when advance warning sign } \\
\text { becomes active. }\end{array}$ \\
\hline 72 & $\begin{array}{l}\text { Advance Warning } \\
\text { Sign Off }\end{array}$ & $\begin{array}{l}\text { Overlap \# (as } \\
\text { number A=1 B=2, } \\
\text { etc) }\end{array}$ & $\begin{array}{l}\text { Set when advance warning sign } \\
\text { becomes inactive. }\end{array}$ \\
\hline
\end{tabular}

Note: Event Codes (EC) 71 and 72 are proposed to log the event when advance warning sign becomes active or inactive. These event codes were proposed by Steve Gault (PennDOT). 
New Event Code 93 and 94

\begin{tabular}{|l|l|l|l|}
\hline Event Code & Event Descriptor & Parameter & Description \\
\hline 93 & TSP Detector Off & TSP \#(1-255) & $\begin{array}{l}\text { TSP detector events shall be } \\
\text { triggered post any detector } \\
\text { delay/extension processing. }\end{array}$ \\
\hline 94 & TSP Detector On & TSP \#(1-255) & \\
\hline
\end{tabular}

Note: Event Codes (EC) 93 and 94 are proposed to log Transit Signal Priority (TSP) detector events. This event code was proposed by Tom Stiles (Intelight Inc.).

New Event Code 116

\begin{tabular}{|l|l|l|l|}
\hline Event Code & Event Descriptor & Parameter & Description \\
\hline 116 & $\begin{array}{l}\text { Preemption Force } \\
\text { Off }\end{array}$ & Preempt \# (1-255) & $\begin{array}{l}\text { Set when preemption force off is } \\
\text { applied to the active cycle. }\end{array}$ \\
\hline
\end{tabular}

Note: Event Code (EC) 116 is proposed to log the event when controller applies preemption force off to the active cycle. This event code is proposed upon the request from Josh Fink (Econolite Group Inc.). Since Event Code 6 (Phase Force Off) is shared by both coordinators and preemption, a new event code eliminates the confusion over the definition of existing EC-6.

New Event Code 117

\begin{tabular}{|l|l|l|l|}
\hline Event Code & Event Descriptor & Parameter & Description \\
\hline 117 & TSP Early Force Off & TSP \#(1-255) & $\begin{array}{l}\text { Set when TSP early force off is } \\
\text { applied to the active cycle. }\end{array}$ \\
\hline
\end{tabular}

Note: Event Code (EC) 117 is proposed to log the event when controller applies early force off to the active cycle during Transit Signal Priority (TSP) operation. This event code was proposed by Tom Stiles (Intelight Inc.). 
New Event Code 120 and 121

\begin{tabular}{|l|l|l|l|}
\hline Event Code & Event Descriptor & Parameter & Description \\
\hline 120 & TSP Service Start & TSP \#(1-255) & $\begin{array}{l}\text { Set when requested TSP service } \\
\text { begins. }\end{array}$ \\
\hline 121 & TSP Service End & TSP \#(1-255) & $\begin{array}{l}\text { Set when requested TSP service } \\
\text { ends. }\end{array}$ \\
\hline
\end{tabular}

Note: Event Codes (EC) 120 and 121 are proposed to log the event when Transit Signal Priority (TSP) service starts and ends. This event code was proposed by Tom Stiles (Intelight Inc.).

New Event Code 152

\begin{tabular}{|l|l|l|l|}
\hline Event Code & Event Descriptor & Parameter & Description \\
\hline 152 & $\begin{array}{l}\text { Coordinated phase } \\
\text { begin }\end{array}$ & Phase \# (1-255) & $\begin{array}{l}\text { Set when coordinated phase } \\
\text { begins. }\end{array}$ \\
\hline
\end{tabular}

Note: Event Code (EC) 152 is proposed to log event when controller begins serving the coordinated phase. This event code was proposed by Dan Nelson (Siemens Industry, Inc.). 


\section{New Event Code 153}

\begin{tabular}{|l|l|l|l|}
\hline Event Code & Event Descriptor & Parameter & Description \\
\hline 153 & $\begin{array}{l}\text { Logic Statement } \\
\text { True }\end{array}$ & $\begin{array}{l}\text { Logic Statement \# } \\
(1-255)\end{array}$ & $\begin{array}{l}\text { Set when the logic statement } \\
\text { evaluation changes from "False" } \\
\text { to "True". }\end{array}$ \\
\hline
\end{tabular}

Note: Event Code (EC) 153 is proposed to log the event when the logic statement evaluation changes from "False" to "True". This event code was proposed by Steve Gault (PennDOT) and Matthew Carlisle (NCDOT).

New Event Code 154

\begin{tabular}{|l|l|l|l|}
\hline Event Code & Event Descriptor & Parameter & Description \\
\hline 154 & $\begin{array}{l}\text { Logic Statement } \\
\text { False }\end{array}$ & $\begin{array}{l}\text { Logic Statement \# } \\
(1-255)\end{array}$ & $\begin{array}{l}\text { Set when the logic statement } \\
\text { evaluation changes from "True" } \\
\text { to "False". }\end{array}$ \\
\hline
\end{tabular}

Note: Event Code (EC) 154 is proposed to log the event when the logic statement evaluation changes from "True" to "False". This event code was proposed by Steve Gault (PennDOT) and Matthew Carlisle (NCDOT). 
New Event Code 155

\begin{tabular}{|c|c|c|c|}
\hline Event Code & Event Descriptor & Parameter & Description \\
\hline 155 & $\begin{array}{l}\text { Unit Control Status } \\
\text { Change }\end{array}$ & $\begin{array}{l}\text { Parameter }(1-10) \\
\text { defined as: } \\
1=\text { Other } \\
2=\text { System } \\
\text { Control } \\
3=\text { System } \\
\text { Standby } \\
4=\text { Backup Mode } \\
5=\text { Manual } \\
6=\text { Timebase } \\
7=\text { Interconnect } \\
8=\text { Interconnect } \\
\text { Backup } \\
9=\text { Remote } \\
\text { Manual Control } \\
10=\text { Local Manual } \\
\text { Control }\end{array}$ & $\begin{array}{l}\text { See NTCIP 1202v0326 } 5.4 .5 \text { for } \\
\text { definition. }\end{array}$ \\
\hline
\end{tabular}

Note: Event Code (EC) 155 is proposed to log the event when control mode for Pattern, Flash, or Free at the controller changes to the parameter (1-10) defined above. This event code was proposed by Steve Gault (PennDOT). 


\section{New Event Code 156}

\begin{tabular}{|c|c|c|c|}
\hline Event Code & Event Descriptor & Parameter & Description \\
\hline 156 & $\begin{array}{l}\text { Additional Cycle } \\
\text { Length Change }\end{array}$ & Seconds (0-255) & $\begin{array}{l}\text { Set simultaneously with Event } \\
\text { Code } 132 \text { when existing cycle } \\
\text { length exceeds } 255 \text { seconds. } \\
\text { When this Event Code } 156 \text { is } \\
\text { used, Event Code } 132 \text { shall be } \\
255, \text { where the value of this } \\
\text { Event Code } 156 \text { will be the } \\
\text { additional cycle length above } 255 \\
\text { (in seconds). Cycle lengths longer } \\
\text { than } 510 \text { seconds will require a } \\
\text { controller look up. }\end{array}$ \\
\hline
\end{tabular}

Note: Event Code (EC) 156 is proposed to log the event when existing cycle length exceeds 255 seconds. When this Event Code 156 is used, Event Code 132 shall be 255, where the value of this Event Code 156 will be the additional cycle length above 255 (in seconds). Cycle lengths longer than 510 seconds will require a controller look up. This event code was proposed by Josh Fink (Econolite Group Inc.).

New Event Code 200 and 201

\begin{tabular}{|l|l|l|l|}
\hline Event Code & Event Descriptor & Parameter & Description \\
\hline 200 & Alarm On & $\begin{array}{l}\text { Alarm \# (as } \\
\text { number A=1 B=2, } \\
\text { etc) }\end{array}$ & $\begin{array}{l}\text { Set when cabinet/system alarm is } \\
\text { activated. }\end{array}$ \\
\hline 201 & Alarm Off & $\begin{array}{l}\text { Alarm \# (as } \\
\text { number A=1 B=2, } \\
\text { etc) }\end{array}$ & $\begin{array}{l}\text { Set when cabinet/system alarm is } \\
\text { released. }\end{array}$ \\
\hline
\end{tabular}

Note: Event Codes (EC) 200 and 201 are proposed to log the event when cabinet or system alarm is activated or released. These event codes were proposed by Donald Maas Jr. (McCain Inc.). 
New Event Code 202

\begin{tabular}{|l|l|l|l|}
\hline Event Code & Event Descriptor & Parameter & Description \\
\hline 202 & Aux Switch On/Off & $\begin{array}{l}\text { Aux switch On/Off } \\
\#(1,0)\end{array}$ & $\begin{array}{l}\text { Set when local controller aux } \\
\text { switch is active (1) or inactive (0) }\end{array}$ \\
\hline
\end{tabular}

Note: Event Codes (EC) 202 is proposed to log the controller auxiliary switch on/off event. This event code was proposed by Dan Nelson (Siemens Industry, Inc.)

New Event Code 203 to 218

\begin{tabular}{|l|l|l|l|}
\hline Event Code & Event Descriptor & Parameter & Description \\
\hline 203 & Split 17 Change & $\begin{array}{l}\text { New Split Time in } \\
\text { Seconds (0-255) }\end{array}$ & $\begin{array}{l}\text { Split change events shall be } \\
\text { populated upon selection of a } \\
\text { new coordination pattern as well } \\
\text { as during a split change to an } \\
\text { active pattern via Signal Control } \\
\text { and Prioritization (SCP), Adaptive } \\
\text { Control System (ACS) Lite or } \\
\text { other adaptive control system. }\end{array}$ \\
\hline 204 & Split 18 Change & $\begin{array}{l}\text { New Split Time in } \\
\text { Seconds (0-255) }\end{array}$ & \\
\hline.. &.. & .. & $\begin{array}{l}\text { New Split Time in } \\
\text { Seconds (0-255) }\end{array}$ \\
\hline 218 & Split 32 Change & & \\
\hline
\end{tabular}

Note: Event Codes $(E C) 203$ to 218 are proposed to log the split change event for extended phase 17-32. Phase limit has been extended from previous 0-16 to proposed 0-32, to provide accommodation for ITS cabinet. These event codes were proposed by Mark Taylor (UDOT). 
Modified Definition - Event Code 4

\begin{tabular}{|c|c|c|c|c|}
\hline Event Code & Event Descriptor & Parameter & Existing Description & Proposed Description \\
\hline 4 & Phase Gap Out & $\begin{array}{l}\text { Phase \# (1- } \\
255)\end{array}$ & $\begin{array}{l}\text { Set when phase gaps } \\
\text { out but may not } \\
\text { necessarily occur } \\
\text { upon phase } \\
\text { termination. Event } \\
\text { may be set multiple } \\
\text { times within a single } \\
\text { green under } \\
\text { simultaneous gap } \\
\text { out. }\end{array}$ & $\begin{array}{l}\text { Phase termination due to } \\
\text { gap out termination } \\
\text { condition. Set once per } \\
\text { phase when phase gaps out } \\
\text { but may not necessarily } \\
\text { occur upon phase } \\
\text { termination. }\end{array}$ \\
\hline
\end{tabular}

Note: The definition of Event Code 4 is modified to eliminate ambiguity in the definition. A new event code (EC 13 - Extension Timer Gap Out) is proposed.

Modified Definition - Event Code 6

\begin{tabular}{|l|l|l|l|l|}
\hline Event Code & Event Descriptor & Parameter & Existing Description & Proposed Description \\
\hline 6 & Phase Force Off & $\begin{array}{l}\text { Phase \# (1- } \\
255)\end{array}$ & $\begin{array}{l}\text { Set when phase } \\
\text { force off is applied to } \\
\text { the active green } \\
\text { phase. }\end{array}$ & $\begin{array}{l}\text { Set when phase force off is } \\
\text { applied by the coordinator } \\
\text { to the green phase. }\end{array}$ \\
\hline
\end{tabular}

Note: The definition of Event Code 6 is modified to eliminate ambiguity in the definition. Since Event Code 6 (Phase Force Off) is shared by both coordinators and preemption, therefore, a new event code is proposed for Preemption Force Off (New Event Code 116) and the existing definition of event code 6 is modified. 
Modified Definition - Event Code 7

\begin{tabular}{|l|l|l|l|l|}
\hline Event Code & Event Descriptor & Parameter & Existing Description & Proposed Description \\
\hline 7 & $\begin{array}{l}\text { Phase Green } \\
\text { Termination }\end{array}$ & $\begin{array}{l}\text { Phase \# (1- } \\
255)\end{array}$ & $\begin{array}{l}\text { Set when phase } \\
\text { green indications are } \\
\text { terminated into } \\
\text { either yellow } \\
\text { clearance or } \\
\text { permissive (FYA) } \\
\text { movement. }\end{array}$ & $\begin{array}{l}\text { Set when phase green } \\
\text { indications are terminated } \\
\text { into either yellow change } \\
\text { interval or permissive (FYA) } \\
\text { movement. }\end{array}$ \\
\hline
\end{tabular}

Note: Event code 7 is modified to keep consistency with the 2009 MUTCD Section 4D.26-Yellow Change and Red Clearance Intervals. This was proposed by Ted Lombardi (Caltrans).

Modified Definition - Event Code 8

\begin{tabular}{|l|l|l|l|l|l|}
\hline $\begin{array}{l}\text { Event } \\
\text { Code }\end{array}$ & $\begin{array}{l}\text { Existing Event } \\
\text { Descriptor }\end{array}$ & $\begin{array}{l}\text { Proposed Event } \\
\text { Descriptor }\end{array}$ & Parameter & $\begin{array}{l}\text { Existing } \\
\text { Description }\end{array}$ & Proposed Description \\
\hline 8 & $\begin{array}{l}\text { Phase Begin } \\
\text { Yellow } \\
\text { Clearance }\end{array}$ & $\begin{array}{l}\text { Phase Begin } \\
\text { Yellow Change }\end{array}$ & $\begin{array}{l}\text { Phase \# (1- } \\
255)\end{array}$ & $\begin{array}{l}\text { Set when phase } \\
\text { yellow indication } \\
\text { becomes active } \\
\text { and clearance } \\
\text { timer begins. }\end{array}$ & $\begin{array}{l}\text { Set when phase yellow } \\
\text { indication becomes } \\
\text { active and interval timer } \\
\text { begins. }\end{array}$ \\
\hline
\end{tabular}

Note: Event code 8 is modified to keep consistency with the 2009 MUTCD Section 4D.26-Yellow Change and Red Clearance Intervals. This was proposed by Ted Lombardi (Caltrans). 
Modified Definition - Event Code 132

\begin{tabular}{|c|c|c|c|c|}
\hline Event Code & Event Descriptor & Parameter & Existing Description & Proposed Description \\
\hline 132 & $\begin{array}{l}\text { Cycle Length } \\
\text { Change }\end{array}$ & $\begin{array}{l}\text { Seconds (0- } \\
255)\end{array}$ & $\begin{array}{l}\text { This event shall be } \\
\text { populated upon } \\
\text { selection of a new } \\
\text { coordination pattern } \\
\text { change that selects a } \\
\text { new cycle length. } \\
\text { Cycle lengths in } \\
\text { excess of } 255 \text { shall } \\
\text { record this event } \\
\text { with a } 255 \\
\text { parameter, requiring } \\
\text { controller database } \\
\text { lookup for this actual } \\
\text { value. }\end{array}$ & $\begin{array}{l}\text { This event shall be } \\
\text { populated upon selection of } \\
\text { a new coordination pattern } \\
\text { change that selects a new } \\
\text { cycle length. Cycle lengths in } \\
\text { excess of } 255 \text { shall record } \\
\text { this event with a } 255 \\
\text { parameter, along with event } \\
\text { code } 156 .\end{array}$ \\
\hline
\end{tabular}

Note: The description of Event Code 132 is modified since a new event code 156 is proposed to log additional cycle length change event. Josh Fink (Econolite Group Inc.) suggested the change.

Modified Definition - Event Code 134

\begin{tabular}{|l|l|l|l|l|}
\hline Event Code & Event Descriptor & Parameter & Existing Description & Proposed Description \\
\hline 134 & Split 1 Change & $\begin{array}{l}\text { New Split } \\
\text { Time in } \\
\text { Seconds (0- } \\
255)\end{array}$ & $\begin{array}{l}\text { Split change events } \\
\text { shall be populated } \\
\text { upon selection of a } \\
\text { new coordination } \\
\text { pattern as well as } \\
\text { during a split change } \\
\text { to an active pattern } \\
\text { via ACS Lite or other } \\
\text { adaptive control } \\
\text { system. }\end{array}$ & $\begin{array}{l}\text { Split change events shall be } \\
\text { populated upon selection of } \\
\text { a new coordination pattern } \\
\text { as well as during a split } \\
\text { change to an active pattern } \\
\text { via Signal Control and } \\
\text { Prionization (SCP), } \\
\text { Adaptive Control System } \\
\text { (ACS) Lite or other adaptive } \\
\text { control system. }\end{array}$ \\
\hline
\end{tabular}

Note: The definition of Event Code 134 is modified since the existing definition only refers to Adaptive Control System (ACS). However, some vendors modify splits with Signal Control and Prioritization (SCP) system. Josh Fink (Econolite Group Inc.) reported this issue. 
Modified Definition - Event Code 150

\begin{tabular}{|c|c|c|c|c|}
\hline Event Code & Event Descriptor & Existing Parameter & Proposed Parameter & Description \\
\hline 150 & $\begin{array}{l}\text { Coord cycle state } \\
\text { change }\end{array}$ & $\begin{array}{l}\text { Parameter (0-6) } \\
\text { defined as: } \\
0=\text { Free } \\
1=\text { In Step } \\
2=\text { Transition - Add } \\
3=\text { Transition - } \\
\text { Subtract } \\
4=\text { Transition - Dwell } \\
5=\text { Local Zero } \\
6=\text { Begin Pickup }\end{array}$ & $\begin{array}{l}\text { Parameter (0-7) } \\
\text { defined as: } \\
0=\text { Free } \\
1=\text { In Step } \\
2=\text { Transition - Add } \\
3=\text { Transition - } \\
\text { Subtract } \\
4=\text { Transition - Dwell } 5 \\
=\text { Local Zero } \\
6=\text { Begin Pickup } \\
7=\text { Master Cycle Zero }\end{array}$ & \\
\hline
\end{tabular}

Note: The parameter of event code 150 is modified with an inclusion of new parameter 7 (Master Cycle Zero). Parameter 7 will be logged when master controller clock turns zero. Modification is proposed by Mark Taylor (UDOT).

Modified Definition - Event Code 151

\begin{tabular}{|l|l|l|l|l|}
\hline Event Code & Event Descriptor & Parameter & Existing Description & Proposed Description \\
\hline 151 & $\begin{array}{l}\text { Coordinated } \\
\text { phase yield point }\end{array}$ & $\begin{array}{l}\text { Phase \# (1- } \\
255)\end{array}$ & & $\begin{array}{l}\text { Set once per cycle for each } \\
\text { coordinated phase when } \\
\text { controller suspends the } \\
\text { coordinated phase. }\end{array}$ \\
\hline
\end{tabular}

Note: The definition of Event Code 151 is added since there was no definition for this event code. 
Modified Definition - Event Code 180

\begin{tabular}{|l|l|l|l|l|}
\hline Event Code & Event Descriptor & Parameter & Existing Description & Proposed Description \\
\hline 180 & $\begin{array}{l}\text { Stop Time Input } \\
\text { On/Off }\end{array}$ & $\begin{array}{l}\text { Stop Time } \\
\text { Input Advance } \\
\text { On/Off \# (1,0) }\end{array}$ & $\begin{array}{l}\text { Set when stop time } \\
\text { input is applied or } \\
\text { removed, regardless } \\
\text { of source of stop. }\end{array}$ & $\begin{array}{l}\text { Set when stop time input is } \\
\text { applied or removed, } \\
\text { regardless of source of stop } \\
\text { or state. }\end{array}$ \\
\hline
\end{tabular}

Note: The definition of Event Code 180 is modified as per suggestion from Ted Lombardi (Caltrans). 